\title{
Research on Traffic Accident Forecasting Based on Gray Model
}

\author{
Tian LingLing, Jiang JunCheng
}

School of safety science and engineering, Nanjing University of Technolog, Nanjing, China

lingling@163.com

Keywords: traffic accident, gray model, forecasting.

\begin{abstract}
As the road traffic system is an uncertain system, the occurrence of traffic accidents is also an uncertain system with partial information known and the other unknown. Therefore, it's suitable to apply the gray model theory to predict the traffic accidents. This paper expounds the principles of gray model and gives an example to show the feasibility and practicability of the gray model applied in the forecasting of traffic accidents.
\end{abstract}

\section{Gray Feature of Traffic Accidents}

Traffic accidents often result in serious consequences, such as casualties, vehicle damage, road congestion and other serious consequences. It's necessary to explore the development trend of road traffic accidents and the development trend of future traffic accidents to prevent and reduce the occurrence of accidents and the loss of traffic accidents. Traffic accident prediction is based on the statistics, analysis and treatment of accident data, and based on the accident causes and development law. Through scientific analysis, we can see that it is not clear that the accident in advance to make logical reasoning. Traffic accident prediction is a key problem in road traffic safety planning, decision making and traffic engineering project benefit evaluation. It has important practical significance to predict the correct or not directly related to the construction of traffic facilities, the formulation of traffic management policies and the allocation of investment funds. Correctly analyzing the characteristics of road traffic accidents and establishing a high quality forecasting model is the key to predict the traffic accidents.

Probability statistics, fuzzy mathematics and gray system theory are the three most commonly used uncertainty systems. Probability statistics mainly uses the regression forecasting model to forecast road traffic accident. The shortcoming of the regression forecasting model is that the establishment of the model needs a lot of traffic accident history data, leaving a large amount of data support, the determination of the equation parameters is very difficult to have the power of persuasion. And the gray prediction method can overcome the above shortcomings, it does not require a large amount of data support, and do not need data to obey the typical probability distribution, even in the case of only a few data can be established to predict the model. The occurrence of road traffic accidents is not obvious. It is a random change. It has the characteristics of random and fuzzy. The occurrence time of traffic accident, the occurrence, the law of the occurrence, the damage is not expected. For random variable, random process, people often use the method of probability statistics. And the method of probability statistics requires that data is large, it must be found out from a large amount of data, and can solve the problem. The gray system theory, then, is considered to be a gray quantity of the change in a certain range, and the random process can be seen as a gray process that changes in a certain range. The gray amount is not from the perspective of statistical rules, through large sample size, but with the data processing method, the chaos of the original data into a more regular pattern of production and then do research. If we consider road traffic in a region as a certain system, the system is gray with some certain factors (white information), such as road conditions, signal signs, etc., and some uncertain factors (black information), such as vehicle condition, climate, driver's psychological state, etc. It can be considered as a gray system which can be used to deal with gray system theory. 


\section{Establishment of Gray Model}

\section{Overview of the Method}

Firstly, we suppose the road traffic accident data sequence is

$$
x^{(0)}=\left\{x_{1}^{(0)}, x_{2}^{(0)}, \ldots, x_{n}^{(0)}\right\} .
$$

We use the gray system theory to establish the GM $(1,1)$ :

$$
x^{(1)}(t+1)=\left[x^{(1)}(1)-\frac{b}{\mu}\right] e^{-\mu t}+\frac{b}{\mu} .
$$

The expression is the predicted values of the generated sequence. However, what we need is the predictive value of the original sequence. Therefore, it's necessary of accumulated generating operation to achieve the predictive value of the original sequence:

$$
\begin{gathered}
x^{(0)}=\left\{a^{(1)} x_{1}^{(1)}, a^{(1)} x_{2}^{(1)}, \ldots, a^{(1)} x_{n}^{(1)}\right\} . \\
\text { In the above expression, } \\
a^{(1)} x_{1}^{(1)}=x_{t}^{(1)}-x_{t-1}^{(1)}=x_{t}^{(0)} .
\end{gathered}
$$

\section{Accuracy Test}

The test of residual error, that is $E(t)$ :

$$
E(t)=\stackrel{\Delta}{x^{(0)}}(t) \cdot x^{(0)}(t) .
$$

The relative residual error is:

$$
e(t)=\frac{E(t)}{x^{(0)}(t)}
$$

The test of posteriori error:

Suppose the mean of the original sequence and the residual errors respectively are

$$
\bar{x}, \bar{E} \text {. }
$$

\begin{tabular}{|c|c|c|}
\hline Level & $\mathrm{P}$ & $\mathrm{C}$ \\
\hline 1 & $>0.95$ & $<0.35$ \\
\hline 2 & $>0.80$ & $<0.45$ \\
\hline 3 & $>0.70$ & $<0.50$ \\
\hline 4 & $<=0.70$ & $<=0.65$ \\
\hline
\end{tabular}

Suppose the mean square error of the original sequence and the residual errors respectively are

Therefore, the ratio of posteriori error is

$$
S_{1}^{2}, S_{2}^{2} \text {. }
$$

The frequency error is

$$
C=\frac{S_{2}}{S_{1}}
$$

$$
P=P\left\{\left|E(t)-\bar{E}<0.6745 * S_{1}\right|\right\}
$$

The accuracy of the gray model prediction can be showed in Table 1.

\section{Empirical Research of Gray Model}

According to the traffic management department of A Province, the number of road traffic accidents in some expressway is increasing year by year from 2006 to 2014. The accident data are shown in Table 2. Now, we forecast the traffic accidents in the next 5 years. 
Table 2 The number of accidents on some expressway between 2006 2014

\begin{tabular}{|l|r|r|r|r|r|r|r|r|r|}
\hline year & 2006 & 2007 & 2008 & 2009 & 2010 & 2011 & 2012 & 2013 & 2014 \\
\hline accidents & 3.98 & 4.02 & 4.1 & 4.16 & 4.24 & 4.36 & 4.46 & 4.55 & 4.65 \\
\hline
\end{tabular}

(Annotation: the unit of accidents is ten thousand.)

So, the original sequence is:

$$
x^{(0)}=\{3.98,4.02,4.1,4.16,4.24,4.36,4.46,4.55,4.65\} .
$$

The generated sequence is:

$$
x^{(1)}=\{3.98,8.01,12.11,20.52,24.89,29.35,33.91\}
$$

Then, we establish the equation:

According to the least square method,

$$
\frac{d x^{(1)}}{d t}+a x^{(1)}=b
$$

$$
B=\left[\begin{array}{cc}
-0.5\left(x^{(1)}(1)+x^{(1)}(2)\right) & 1 \\
-0.5\left(x^{(1)}(2)+x^{(1)}(3)\right) & 1 \\
\vdots & \vdots \\
-0.5\left(x^{(1)}(n-1)+x^{(1)}(n)\right) & 1
\end{array}\right]
$$

Then, we use the Matlab to get $a=-0.0212, b=388.1$.

So the formula of the forecasting of traffic accidents is:

$$
\left\{\begin{array}{l}
\widehat{x}^{(1)}(k+1)=187.05 e^{0.0212 k}-183.06(k=0,1,2) \\
\widehat{x}^{(0)}(k+1)=\widehat{x}^{(1)}(k+1)-\widehat{x}^{(1)}(k) \\
\widehat{x}^{(0)}(1)=\widehat{x}^{(1)}(1)
\end{array}\right.
$$

We can see the fitting effective from the Table 3.

Table 3 Fitting Effective of Gray Model

\begin{tabular}{|c|r|r|r|}
\hline year & original value & \multicolumn{1}{l|}{ fitting value } & error \\
\hline 2006 & 3.98 & 3.98 & 0 \\
\hline 2007 & 4.02 & 4 & 0.02 \\
\hline 2008 & 4.1 & 4.09 & 0.01 \\
\hline 2009 & 4.16 & 4.18 & -0.02 \\
\hline 2010 & 4.24 & 4.27 & -0.03 \\
\hline 2011 & 4.36 & 4.36 & 0 \\
\hline 2012 & 4.46 & 4.45 & 0.01 \\
\hline 2013 & 4.55 & 4.55 & 0 \\
\hline 2014 & 4.65 & 4.64 & 0.01 \\
\hline
\end{tabular}

Then, we can achieve

$$
\begin{gathered}
C=\frac{S_{2}}{S_{1}}=0.054<0.35 \\
P=P\left\{\left|E(t)-\bar{E}<0.6745 * S_{1}\right|\right\}=1>0.95
\end{gathered}
$$

According to Table 1, the accuracy level is 1 .

The forecasting value is showed in Table 4.

\begin{tabular}{|c|r|}
\hline year & traffic accidents \\
\hline 2015 & 4.74 \\
\hline 2016 & 4.85 \\
\hline 2017 & 4.95 \\
\hline 2018 & 5.06 \\
\hline 2019 & 5.16 \\
\hline
\end{tabular}

(Annotation: the unit of accidents is ten thousand.) 


\section{Conclusion}

By analyzing the characteristics of road traffic, we know that the road traffic system can be regarded as a dynamic system. The traffic accident is the result of many kinds of factors in the road traffic system. Gray system theory can solve this problem effectively. In the process of modeling, it advocates respect for original data and not rigidly adhere to the original data, and allows for the analysis of scientific qualitative analysis. In this paper, the gray model of road traffic accidents is established, and the model accuracy of the model is proved to be accurate. gray system theory has been used to build a model, and the model has a dynamic continuity. Over the past ten years, gray theory has been widely used in the forecasting of economic, agricultural, military, ecology, meteorology, and many other areas. The above results show that the accuracy of the method is acceptable, and the forecasting is credible.

\section{References}

[1] Liang Yali, The Analysis of Method in Traffic Accident Forecasting, J., Shanxi Science and Technology, 2(2006)99-101.

[2] Han Wentao, Zhang Qian, Jia Anmin, Study on the forecast model of road accident with gray theory, J., Xi'an University of Arch. \& Tech. (Natural Science Edition), 3(2009)375-377.

[3] Liu Zhaohui, Wang Chao, Xu Hongguo, gray Prediction Model for Road Traffic Accident and Its Application Research, J., Journal of Changchun University of Science and Technology, 4(2006)42-46.

[4] Liu Li, Study on Statistic Analysis and Prediction Model of Road Traffic Accident, D, Chongqing University, 2014. 